\title{
DNA binding activities of the Herves transposase from the mosquito Anopheles gambiae
}

\author{
Amandeep S Kahlon', Robert H Hice, ${ }^{2}$ David A O'Brochta ${ }^{3}$ and Peter W Atkinson 1,2*
}

\begin{abstract}
Background: Determining the mechanisms by which transposable elements move within a genome increases our understanding of how they can shape genome evolution. Class 2 transposable elements transpose via a 'cut-andpaste' mechanism mediated by a transposase that binds to sites at or near the ends of the transposon. Herves is a member of the $h A T$ superfamily of class 2 transposons and was isolated from Anopheles gambiae, a medically important mosquito species that is the major vector of malaria in sub-Saharan Africa. Herves is transpositionally active and intact copies of it are found in field populations of $A$ gambiae. In this study we report the binding activities of the Herves transposase to the sequences at the ends of the Herves transposon and compare these to other sequences recognized by hAT transposases isolated from other organisms.

Results: We identified the specific DNA-binding sites of the Herves transposase. Active Herves transposase was purified using an Escherichia coli expression system and bound in a site-specific manner to the subterminal and terminal sequences of the left and right ends of the element, respectively, and also interacted with the right but not the left terminal inverted repeat. We identified a common subterminal DNA-binding motif (CG/AATTCAT) that is critical and sufficient for Herves transposase binding.

Conclusions: The Herves transposase binds specifically to a short motif located at both ends of the transposon but shows differential binding with respect to the left and right terminal inverted repeats. Despite similarities in the overall structures of $h A T$ transposases, the regions to which they bind in their respective transposons differ in sequence ensuring the specificity of these enzymes to their respective transposon. The asymmetry with which the Herves terminal inverted repeats are bound by the transposase may indicate that these differ in their interactions with the enzyme.
\end{abstract}

\section{Background}

Transposable elements (TEs) are ubiquitous components of genomes in which they impact genomic evolution and maintenance [1-6]. Their mobility properties have resulted in their adoption as genetic tools in modern genetics with one of their many uses in biotechnology being the introduction of foreign genes into insect disease vectors of medical and agricultural importance [7-14]. Anopheles gambiae is the principal vector of the malaria-causing parasite Plasmodium falciparum in subequatorial Africa and is a mosquito species in which robust TE-based genetic tools need to be developed. At present there are six reports of successful genetic

\footnotetext{
* Correspondence: peter.atkinson@ucr.edu

'Interdepartmental Graduate Program in Cell, Molecular and Developmental Biology, University of California, Riverside, CA, USA

Full list of author information is available at the end of the article
}

transformation of this mosquito, one using the $P$ element, and five using the piggyBac element, transformation remaining a low frequency event [9,15-19]. Isolating active, well adapted, endogenous TEs from $A$ gambiae and understanding their biology is likely to improve the efficiency of genetic transformation in this species since these native active TEs are likely to have adapted to overcome or evade the host response systems that are proposed inactivate mobile DNA [20,21].

Herves is an active class 2 TE that was isolated from $A$ gambiae [22]. It contains a transposase-encoding open reading frame (ORF) that is flanked by left (Herves-L) and right (Herves-R) end sequences with the Herves- $\mathrm{L}$ end being unusually long (1,478 bp) compared with the Herves-R end (421 bp) and contains three 100 bp imperfect tandem repeats, commencing $146 \mathrm{bp}$ from the end (Figure 1a). Herves has $11 \mathrm{bp}$ imperfect terminal 
inverted repeats (TIRs) at the left (L-TIR) and right (RTIR) ends (Figure 1a) [22]. It is transpositionally active and can genetically transform Drosophila melanogaster [22]. Population dynamics studies suggest that Herves has been recently active within field populations of $A$ gambiae from Kenya and that many intact copies of it are present in these populations [23]. Class 2 TEs often accumulate internal deletions over time that render the elements inactive and so unable to cause further harm to the host organism [24]. The presence of intact forms of Herves and other hAT TEs, such as Hermes, indicate that at least some $h A T$ elements appear, for reasons unknown, less prone to accumulating internal deletions however the significance of this in absence of information concerning MITEs (Miniature Inverted Terminal Elements) generated from them remains unknown $[23,25,26]$.

Class 2 transposases typically bind to the TIRs and nearby internal sequences and mediate transposition to a new genomic location by the classical 'cut-and-paste'

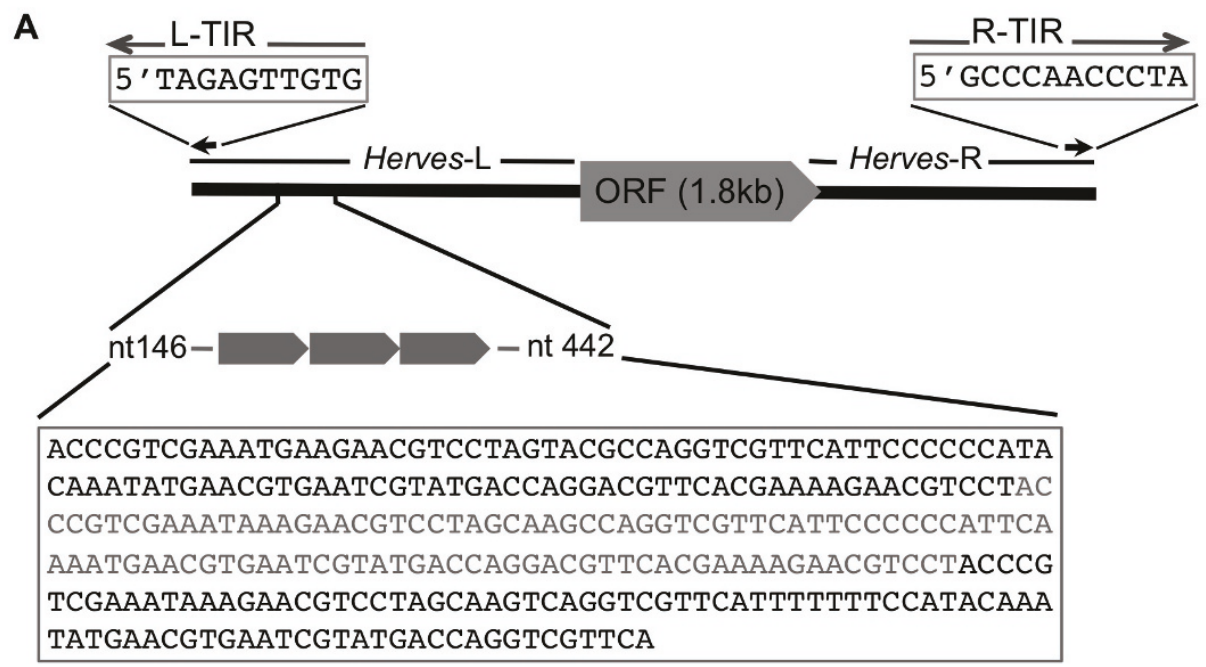

B

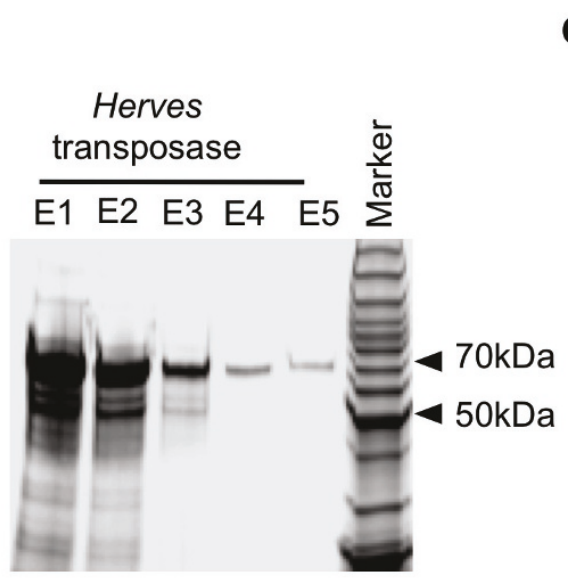

C

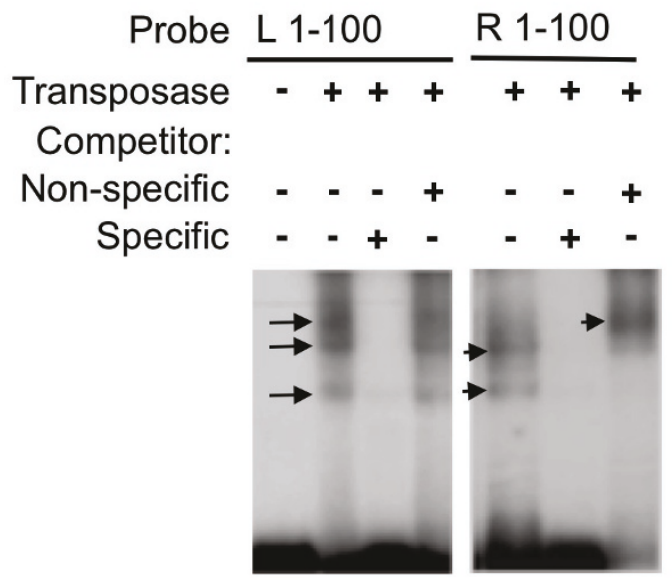

Figure 1 Herves transposase binds to the terminal sequences of $\mathbf{L}$ and $\mathbf{R}$ ends of the Herves element. (a) Schematic representation of the Herves element. Numbers indicate the distance in bp internal to either the left (L) or right (R) end. (b) SDS-PAGE analysis of purified Herves transposase. A Coomassie-stained gel shows $70 \mathrm{kDa}$ purified Herves transposase. E1-E5 represent different elutions obtained during the final step of protein purification. (c) Transposase binding to the terminal fragment of Herves- $L$ and Herves-R ends. Electrophoretic mobility shift assay (EMSA) analysis with the Herves-L bp 1-100 (lanes 1-4) and Herves-R bp 1-100 (lanes 5-7) probes. The DNA fragments were incubated in the presence (+) or absence (-) of pure transposase. A homologous fragment was used as specific competitor. The E1 flanking sequence from the Hermes transposable element was used as the non-specific competitor [44]. Specific and non-specific competitors were use at 200-fold molar excess to the probe. Arrows indicate various protein DNA complexes. 
mechanism [27]. Other cis-acting sequences, which usually consist of short repeat sequence motifs located close to the TIRs, are also important for proper transposase binding and efficient excision and transposition [28-35]. In many cases, native cis elements are not optimized for maximal transposition mobility; thus, new and improved TE gene vectors can be designed by altering these elements to increase or decrease transposase binding $[36,37]$. The identification and characterization of these transposase binding sites and of the specific DNAbinding transposase residues is therefore important to our understanding of the biology and post integration behavior of TEs. This study aimed to identify the DNA sequences of the Herves element bound by its transposase.

\section{Results \\ Purification of Herves transposase and its binding to the Herves-L end}

Herves transposase is 603 amino acids in length and is predicted to have a molecular weight of $70 \mathrm{kDa}$. Herves transposase was purified from an Escherichia coli expression system and its size was confirmed by SDSPAGE (Figure 1b). To examine the binding of Herves transposase to the Herves-L end, we focused on the terminal 100 bp region. A radioactively labeled Herves-L 1-100 bp probe was incubated in the presence or absence of purified Herves transposase for use with a molar excess (200-fold) of unlabeled specific and nonspecific DNA fragments were used as specific and nonspecific competitors, respectively, in electrophoretic mobility shift assays (EMSAs). The transposase interacted with the Herves-L 100 bp probe and formed three transposase-DNA complexes (Figure 1c). A specific competitor competed for the transposase, but the nonspecific competitor did not affect binding (Figure 1c) implicating a sequence-specific interaction between the transposase and the probe.

To specify the transposase binding site(s) within this terminal 100 bp sequence, overlapping oligonucleotides (approximately $30 \mathrm{bp}$ in length) were competed with the Herves-L 100 bp probe for transposase binding. The DNA fragments Herves-L bp 12-48 and bp 28-60 competed with this probe in all three transposase-DNA complexes, whereas the Herves-L bp 1-30, bp 48-75, and bp 76-100 fragments had no effect (Figure 2a). This suggested that the Herves transposase binds tightly and specifically within the L bp 12-60 region. The overlapping Herves-L bp 1-30 and bp 48-75 fragments did not alter binding, indicating that a binding motif(s) was present in the Herves-L bp 28-48 bp (Figure 2a). We also observed that the L bp 12-48 bp and bp 28-60 fragments competed partially with the 100 bp probe, whereas the specific bp 1-100 fragment competed fully for transposase binding, implicating the existence of additional binding motifs that act cooperatively with the binding motif(s) in the bp 28-48 region (Figure 2a).

To confirm these results, each of the unlabeled $30 \mathrm{bp}$ fragments was tested against the Herves-L bp 12-48 probe for binding to the transposase. Binding to the transposase was observed for this probe (Figure 2b), which resulted in two transposase-DNA complexes. The unlabeled L bp 28-60 fragment specifically competed for binding of the transposase (Figure 2b). Herves-L bp 4875 , bp 61-90, and bp 76-100 competed partially with the probe, indicating weak transposase binding to these regions. These results suggested that Herves-L bp 12-48 and bp 28-60 have strong and equal binding for Herves transposase, leading us to believe that the DNA binding motif lay within the Herves-L bp 28-48 fragment.

We performed DNase I protection assays to confirm the EMSA results and to specifically identify the DNA region bound by pure transposase. A terminal 1-100 bp fragment was labeled at the 3' end and labeled probes were incubated separately with Herves transposase and subsequently with DNase I and then analyzed on a denaturing polyacrylamide gel. The two 3' end-labeled probes were protected at bp 25-73 and bp 30-75, respectively (Figure 3 ). Increasing amounts of transposase led to greater protection of the Herves-L $100 \mathrm{bp}$ probe (Figure 3). Overall, the DNase I protection assay results confirmed the EMSA findings, indicating sequence-specific binding of transposase to at least the Herves-L bp 28-48 region.

\section{Transposase binds to the Herves- $\mathrm{R}$ end}

To investigate the binding of transposase to the Herves$\mathrm{R}$ end, the Herves-R 1-100 bp fragment was radiolabeled and used in EMSAs. Herves transposase interacted specifically with the probe and formed two transposaseDNA complexes (Figure 1c). Unlabeled specific competitor competed with the probe for transposase and, notably, the addition of a non-specific competitor led to the formation of a single, higher-molecular-weight complex (Figure 1c). The molecular composition of this complex, however, is unknown.

Overlapping $30 \mathrm{bp}$ oligonucleotides were then used as probes to identify the transposase binding site(s) within the Herves-R 1-100 bp region by EMSA. The bp 1-30, bp 15-45, and bp 61-90 fragments elicited specific binding of transposase (Figure 4a). Fragment bp 31-60 showed weak, non-specific binding, whereas the bp 4675 and bp 91-110 fragments failed to bind (Figure 4a).

Two transposase-DNA complexes formed with the Herves- $\mathrm{R}$ bp 1-30, compared with a single complex each with the Herves-R bp 15-45 and bp 61-90 fragments, implicating the existence of two transposase binding sites within Herves- $\mathrm{R}$ bp 1-30 fragment and one site 


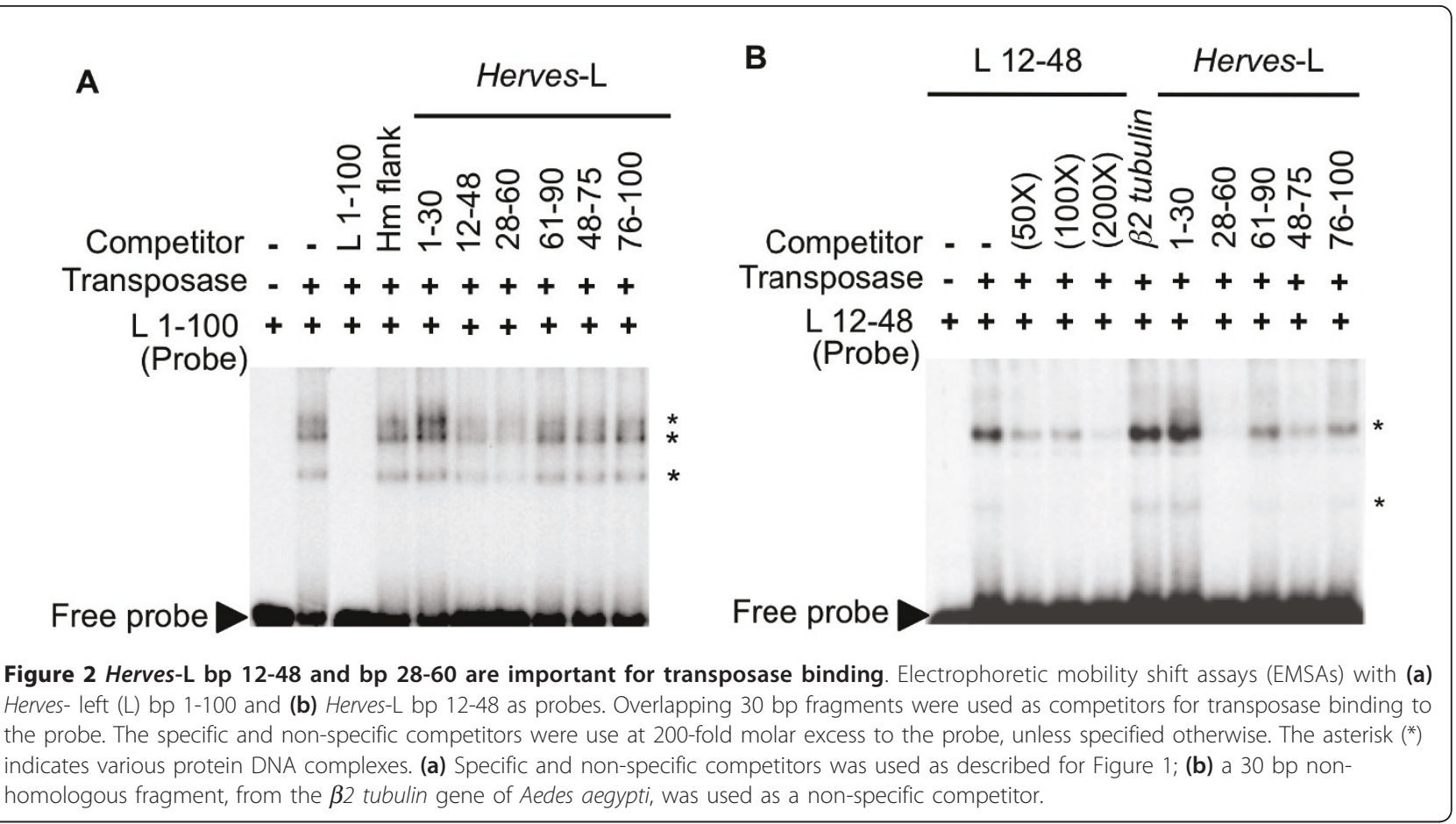

within both the Herves-R bp 15-45 and bp 61-90 fragments (Figure 4a). To determine relative transposase binding preferences, each $30 \mathrm{bp}$ overlapping DNA fragment was allowed to compete against the Herves- $\mathrm{R}$ bp 61-90 probe for transposase binding using EMSAs. Fragment bp 15-45 successfully competed against the probe for transposase, whereas the Herves- $\mathrm{R}$ bp 1-30 and bp 31-60 fragments had no effect (Figure 4b). These data suggest that the transposase binds strongly to the terminal Herves-R end at positions bp 15-45 and bp 61-90.

We performed DNase I protection assays to identify specific binding motifs in the $\mathrm{R}$ end of Herves however these were inconclusive and showed some evidence of protection at bp 23-35 and bp 63-92 (Figure 4c).

\section{Mutational analysis of the Herves transposase binding motif}

Because the Herves-L bp 28-48 fragment showed the strongest binding to transposase, a detailed analysis was performed to define the critical nucleotides for binding. We analyzed 22 sequence variants for their ability to compete with the Herves-L bp 28-60 probe for transposase. Each sequence variant differed from the wild-type sequence by a single nucleotide. An unlabeled wild-type Herves-L bp 28-60 fragment competed successfully against the probe for transposase binding, whereas mutating nucleotides Herves-L bp 32-36 and bp 43-45 abolished this competition, indicating that nucleotides at these positions mediate the binding of transposase (Figure 5a). We identified a conserved binding motif,
CG/AATTCAT, in both regions, suggesting that it constitutes the transposase-binding motif. To confirm these results, we simultaneously mutated this putative motif at both locations within the Herves-L bp 28-60 fragment and allowed the mutant (Herves-L 31-47mut) to compete against the wild-type Herves-L bp 28-60 probe. Mutating both sites abolished the interaction, confirming that CG/AATTCAT is the binding site for Herves transposase in the Herves-L end (Figure 5b).

The CG/AATTCAT motif is conserved between the Herves$L$ and Herves- $R$ ends

We identified similar potential binding motifs within the Herves-R bp 15-22 and bp 73-86 regions. Furthermore, the bp 1-30 region also contains the R-TIR, a potential candidate for transposase binding. To determine whether the R-TIR or the CG/AATTCAT motif mediated the binding of transposase to the Herves-R bp 1-30 region, we mutated each region (Herves- $\mathrm{R}$ TIRmut and Herves- $\mathrm{R}$ bp 15-22mut) and subjected them to EMSA. Mutating each potential binding site abolished its ability to compete against the wild-type probe, suggesting that the CG/ AATTCAT motif and R-TIR are both important for the transposase binding to the Herves-R end (Figure 5c).

\section{The CGATTCAT motif is sufficient for purified Herves transposase binding}

The CG/AATTCAT motif and its derivatives are repeated several times within the transposase binding regions at both the Herves-L and Herves- $\mathrm{R}$ ends (Figure 6). 


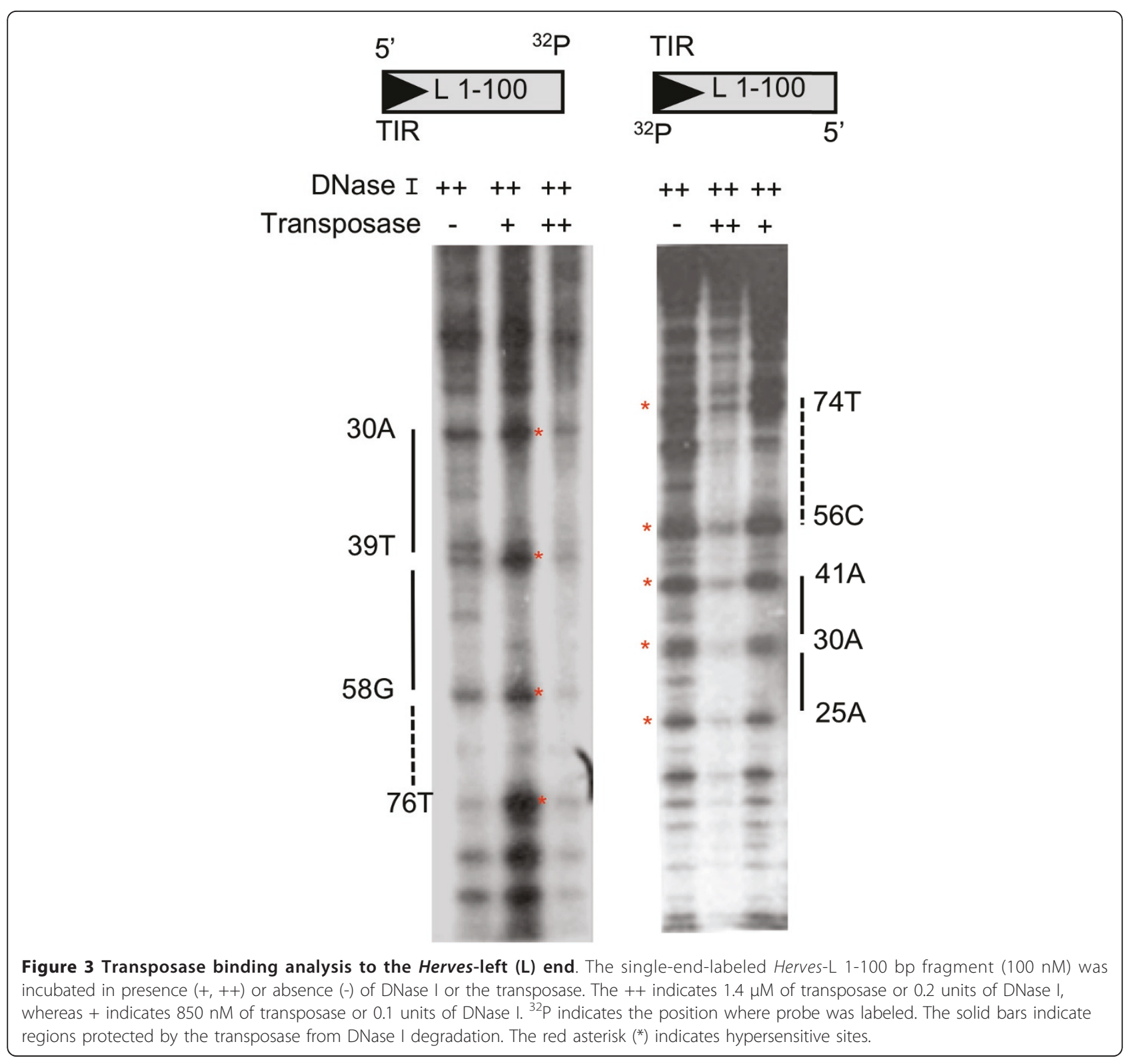

To determine whether the CG/AATTCAT motif was sufficient for transposase binding, we used a probe containing four direct repeats of the CGATTCAT sequence as a probe to measure relative binding to the Herves transposase. The transposase bound to the (CGATTCAT) $)_{4}$ probe and formed two transposase-DNA complexes (Figure 7). Based on the unlabeled specific and non-specific competitors, the interaction was determined to be sequence specific.

We used Herves-L bp 28-60 as a specific competitor for transposase against the $(\mathrm{CGATTCAT})_{4}$ probe and found that it outcompeted it for transposase (Figure 7). Furthermore, splitting the CGATTCAT motif in half abolished the binding (data not shown). Together, these data indicated that the CGATTCAT motif was sufficient for the transposase binding.

We also tested the ability of unlabeled sequence variants of the CGATTCAT motif (CGATTCTT/CGATTCAC/CGTTCAT) to compete against radiolabeled CGATTCAT for transposase binding. None of the sequence variants competed fully with CGATTCAT for the transposase, indicating that CGATTCAT is the strongest binding motif (Figure 7). Nevertheless, CGATTCAC competed partially for transposase, suggesting that this variant may also be important for binding of transposase. 
A

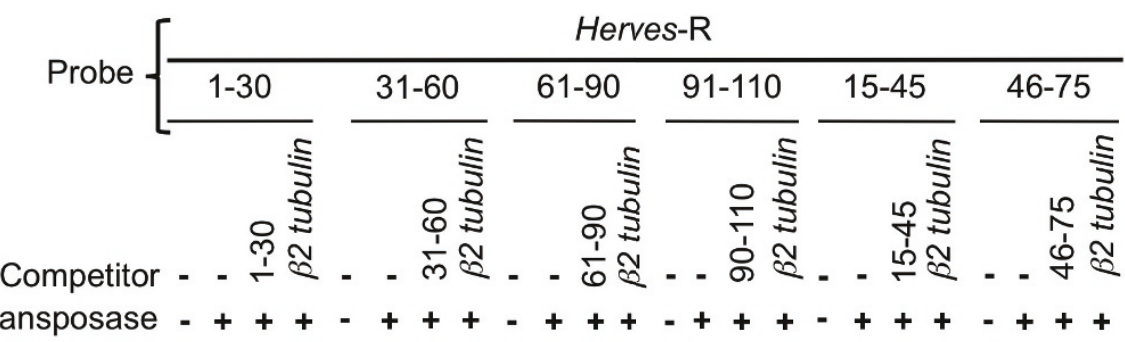

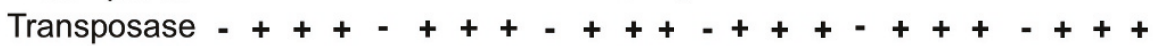

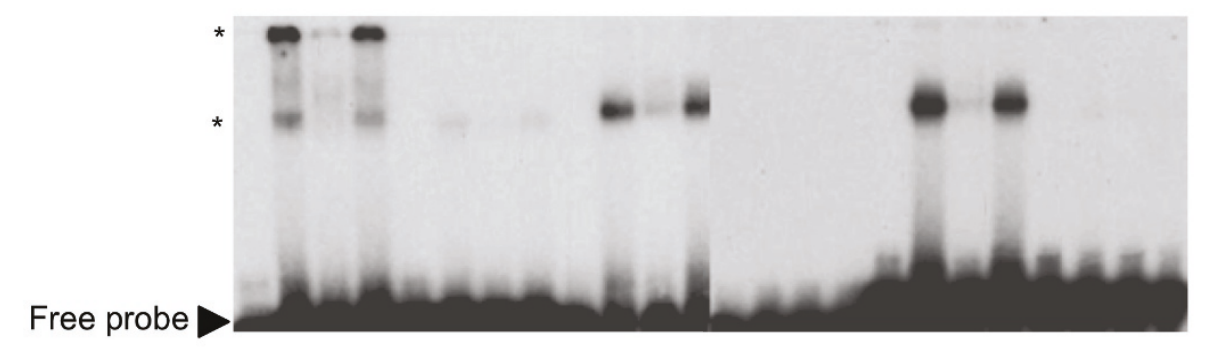

B

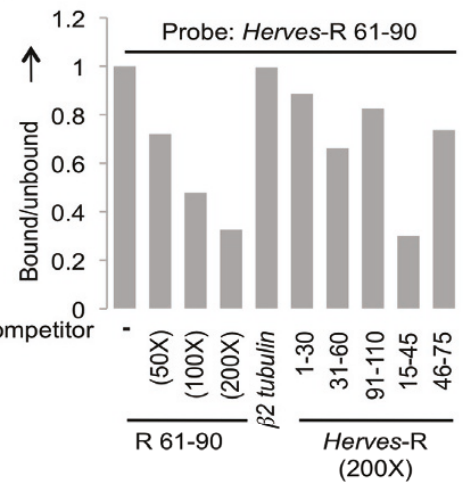

C
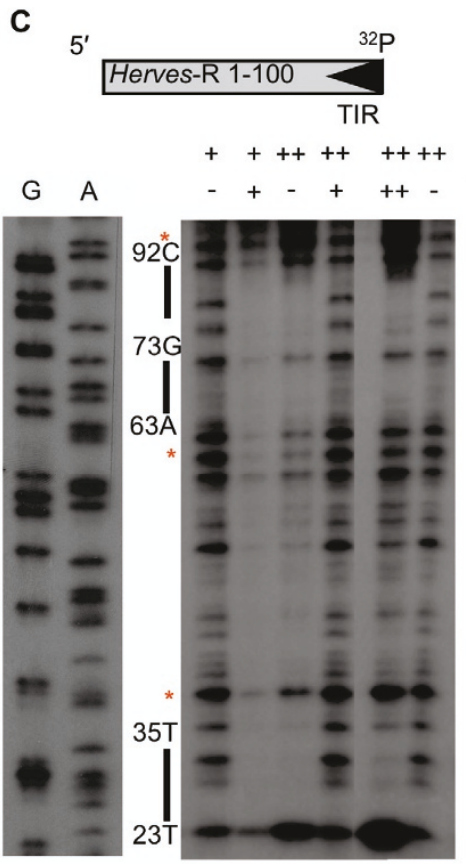

Figure 4 Transposase binding to the Herves-right (R) end. (a) Herves transposase binds to Herves-R bp 1-30, bp 5-45 and bp 61-90. Electrophoretic mobility shift assay (EMSA) analysis of transposase binding to the overlapping 30 bp fragments (bp 1-30, bp 31-60, bp 61-90, bp 91-110, bp 15-45 and bp 46-75). The asterisk $\left(^{*}\right)$ indicates various protein DNA complexes. (b) Herves transposase binding to Herves-R bp 15-45 and $\mathrm{bp}$ 61-90. The Herves-R bp 61-90 fragment was used as a probe in EMSAs. The fraction of the transposase-bound probe was quantified using a phosphoimager. A homologous fragment was used as specific competitor at a molar excess of 50-fold, 100-fold and 200-fold, whereas non-specific competition was used as described for Figure 2b. Overlapping fragments (bp 1-30, bp 31-60, bp 91-110, bp 15-45, and bp 46-75) were used as competitors of transposase binding to the probe, at 200-fold molar excess. (c) DNase I protection assay of the Herves-R end. The single-end-labeled Herves-R 1-100 bp fragment (100 nM) was incubated in presence $(+,++)$ or absence $(-)$ of DNase I or the transposase. The ++ indicates $1.4 \mu \mathrm{M}$ of transposase or 0.5 units of DNase I, whereas + indicates $850 \mathrm{nM}$ of transposase or 0.25 units of DNase I. ${ }^{32} \mathrm{P}$ indicates the end of the probe that was labeled. The solid bars on the sides indicate the region of the probe protected by the transposase. The asterisk (*) indicates hypersensitive sites. 


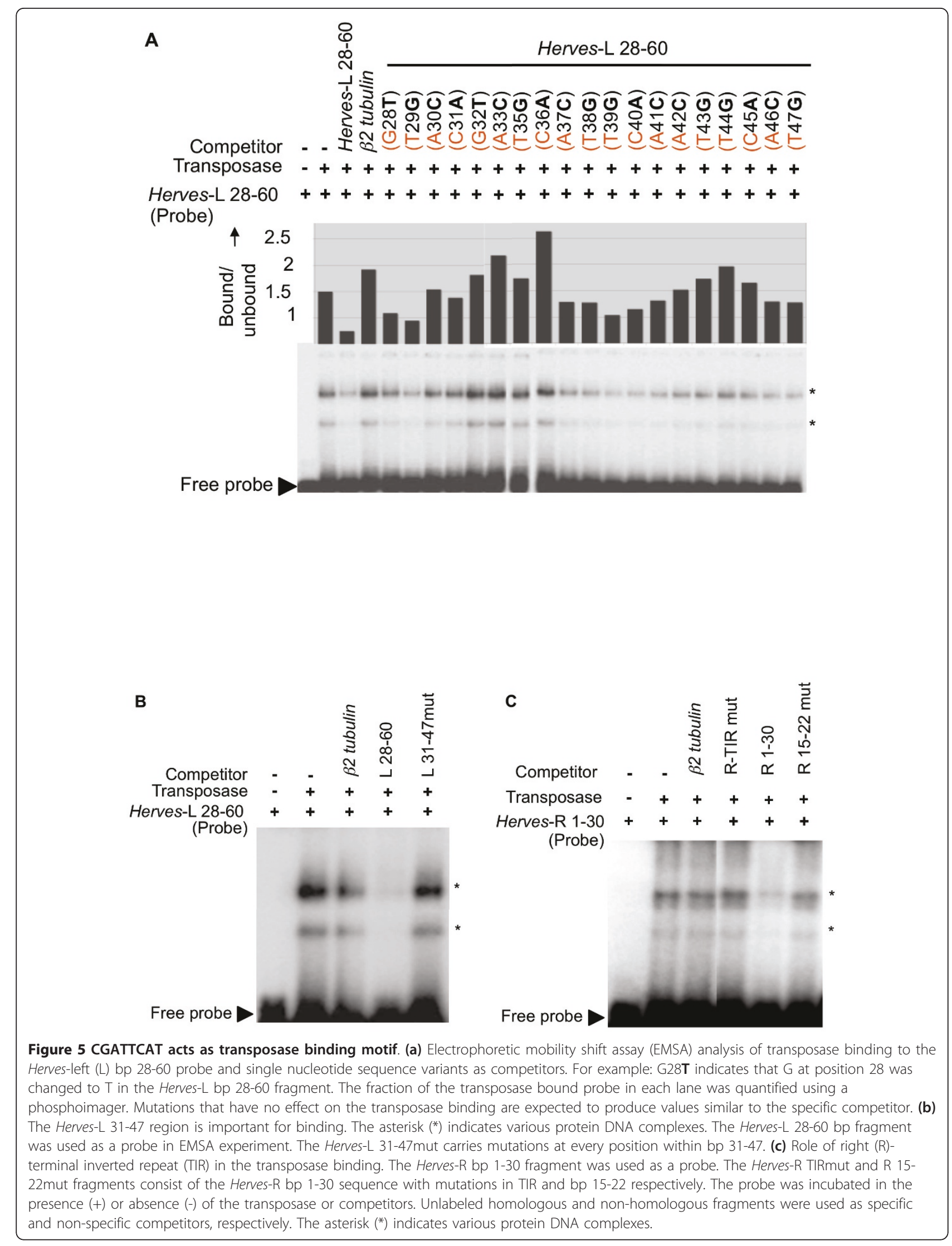



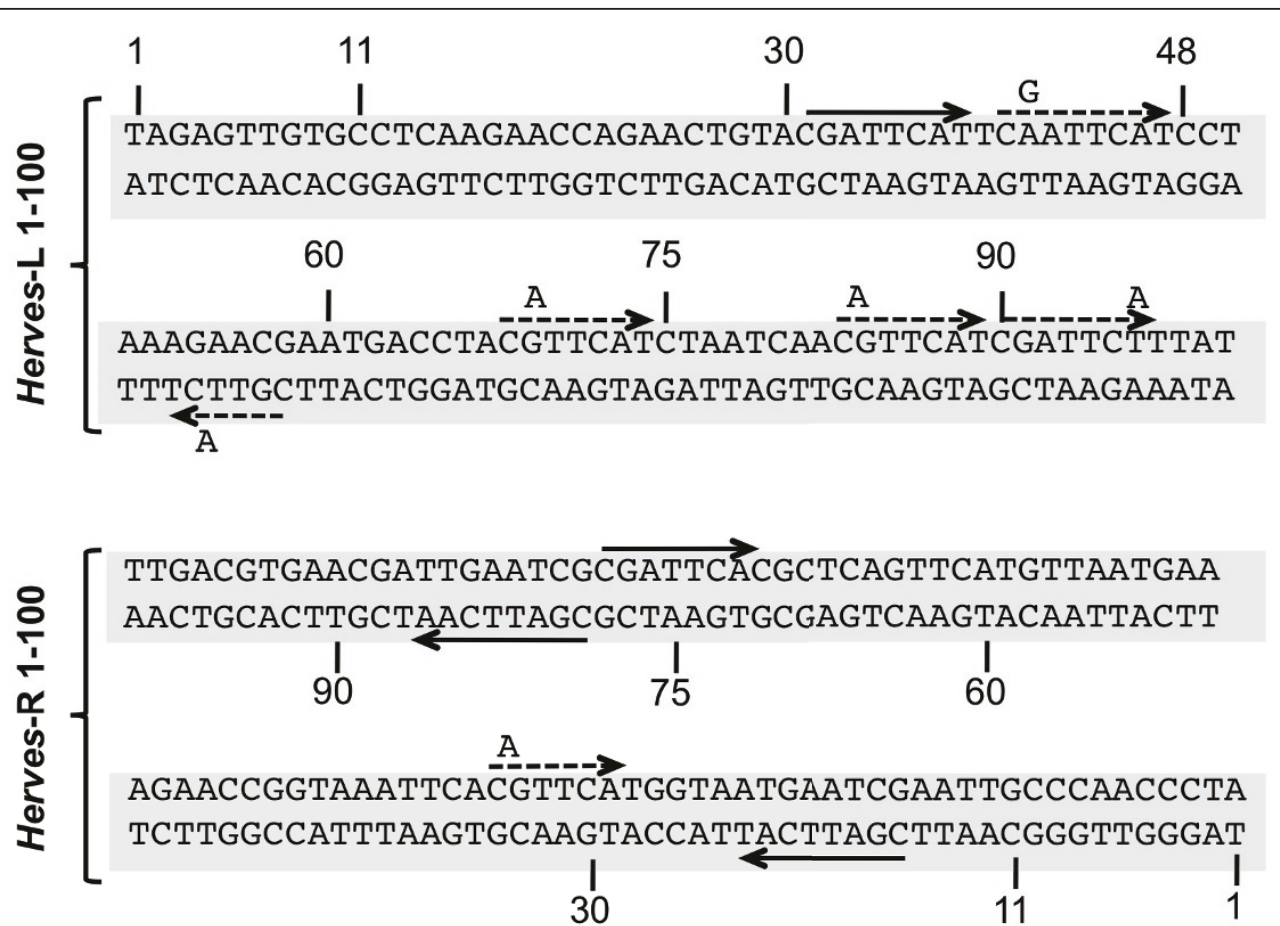

Figure 6 The CGATTCAT binding motif is sufficient for transposase binding. The probe (CGATTCAT) 4 represents four direct repeats of the CGATTCAT sequence motif. Unlabeled (CGATTCAT) 4 and Herves-left (L) bp 28-60 were used as specific competitors, whereas $\beta 2$ tubulin was used as the non-specific competitor. The transposase binding was compared between the sequence variants of the binding motif such as CGATTCTT, CGATTCAC and CGTTCAT (each used as four direct repeats). The asterisk $\left(^{*}\right)$ indicates various protein DNA complexes.

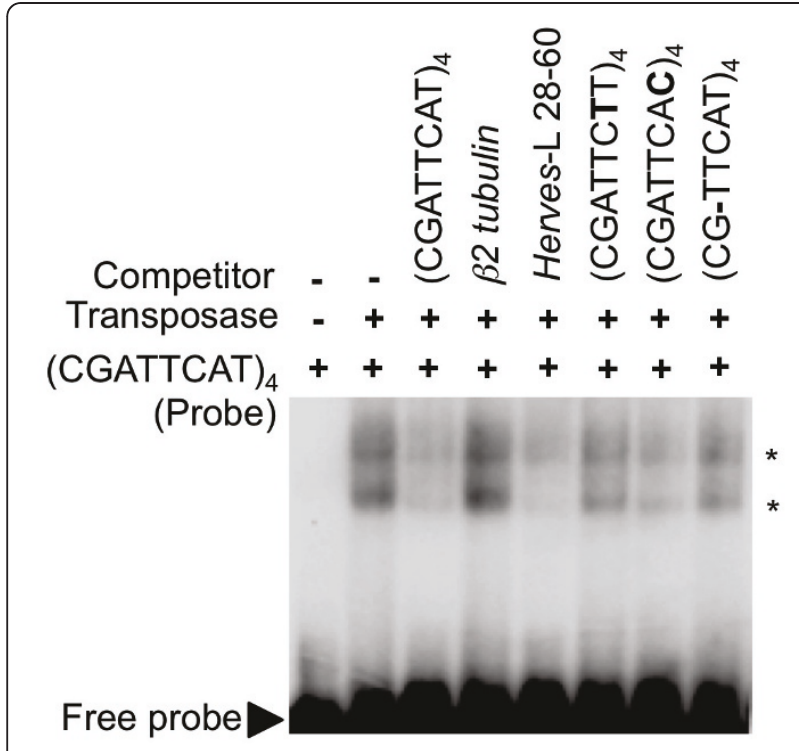

Figure 7 Sequence of Herves-left (L) bp 1-100 and Hervesright $^{\circledR}$ bp 1-100 showing sequence repeats. The solid arrow indicates conserved CGATTCA transposase binding motif, whereas the dotted arrow indicates the single nucleotide sequence variants.

\section{Discussion}

We purified active Herves transposase and demonstrated that it site-specifically binds to subterminal and terminal sequences at the Herves- $\mathrm{L}$ and Herves- $\mathrm{R}$ ends, respectively. Such asymmetrical binding may affect transposition frequency. The Drosophila $P$ element transposase has been shown to bind asymmetrically to the $P$ ends and interchanging the $\mathrm{L}$ end sequence with the $\mathrm{R}$ end sequence led to fewer transposition events [38]. This phenomenon also occurs for the Ac element in maize and the Tag1 element in Arabidopsis [28,31].

There was strong transposase binding to the Herves- $\mathrm{L}$ bp 12-48 and bp 28-60 regions and relatively weak binding to bp 48-75 as shown by EMSA and DNase I footprinting. None of these fragments however, outcompeted the L bp 1-100 probe for transposase binding, suggesting that the binding was cooperative between two or more regions. Furthermore, the overlapping Herves-L bp 12-48 and bp 28-60 fragments showed similar levels of binding, indicating that the binding motif lies in the overlapping region in Herves-L bp 2848. In contrast to the $\mathrm{L}$ end, the binding occurred 
toward the terminal sequences on the Herves- $\mathrm{R}$ end in regions bp 15-45 and bp 61-90.

EMSA results with the Herves-L bp 28-60 probe and single nucleotide sequence variants indicated that the CGATTCAT motif, or its derivatives, mediated binding of the transposase. The CGATTCAT transposase-binding motif and its derivatives are repeated and conserved in the Herves- $\mathrm{L}$ and Herves- $\mathrm{R}$ end sequences. Our results suggested that this motif is important and sufficient for transposase binding, because: (1) mutating the CGATTCAT motif at either end abolished binding, and (2) the transposase bound specifically to a synthetic tetramer of the motif.

TEs frequently have multiple transposase binding sites adjacent to their TIRs [33,39-42]. In other $h A T$ elements, such as Ac, Tol2 and Tag1, their respective transposases bind to short sequence repeats $[31,33,34,43]$. For Ac and Tol2, the transposase binding sequence motifs differ at the $\mathrm{L}$ and $\mathrm{R}$ ends $[33,34]$. The Herves transposase-binding CGATTCAT motif, however, is highly conserved at both ends with several single nucleotide variants CGATTCAC, CGTTCAT, and CGATTCTT being present. Our results suggest that these additional motifs may also mediate transposase binding. Although these derivatives are related to the CGATTCAT motif, their ability to bind transposase differs. The transposase binds to CGATTCAT, but weakly to the CGATTCAC and CGTTCAT motifs. It is also possible that the transposase only recognizes a subset or a family of related sequences in which GATTC or ATTCA is the central sequence. Similar results have been reported for the Tag1 element, for which the R-TGACCC and LAAACCC motifs have different affinities for the transposase $[31,43]$. The sequences that flank these motifs differ, and although they might fail to influence transposase binding, they may regulate transposition [31].

We observed no binding to the L-TIR. Several related $h A T$ transposases, such as $A c$ and Tagl, do not bind their L-TIR and R-TIR sequences [33,43]. This phenomenon raises the possibility that transposase binding to the L-TIR may require the presence of a host factor however nuclear extracts from a Herves transposaseexpressing Drosophila S2 cell line did not bind to L-TIR making the argument for such a factor less compelling. Nevertheless, pure Herves transposase interacted with the R-TIR sequence, the binding at which appeared to be cooperative since both the R-TIR and CGATTCAT motif at 15-22 bp participated in it.

We have identified the sites within the Herves element to which the Herves transposase binds and shown that it binds asymmetrically to sequences at either end of the element. Future work will be directed towards determining whether mutants of Herves which show changes in the binding of the transposase will affect the transpositional activity of Herves in vivo leading to the development of this endogenous TE of $A$ gambiae as a genetic tool in this medically important mosquito species.

\section{Conclusions}

We identified the specific DNA-binding sites of the Herves transposase, a member of the $h A T$ transposon superfamily. We found that it displayed an asymmetry of specific binding to the $\mathrm{L}$ and $\mathrm{R}$ ends of the Herves transposon in that it bound to both subterminal regions but interacted only with the R, but not L, TIR. We identified a common subterminal DNA-binding motif (CG/ AATTCAT) that is critical and sufficient for Herves transposase binding. The asymmetry of binding of the transposase to the $\mathrm{L}$ and $\mathrm{R}$ ends may indicate that these ends differ in their interactions with the enzyme during the transposition reaction. The differences in transposase binding sites between different $h A T$ transposases illustrates that this superfamily provides a fascinating diversity with which to study the biology of transposition.

\section{Methods}

\section{Plasmid constructions}

The Herves ORF was cloned into pBAD myc/HisA (Invitrogen, Carlsbad, CA). The BspHI (incorporated into the Herves start codon) and $K p n \mathrm{I}$ restriction sites were used to amplify a 766-bp fragment of the Herves ORF using the HervesF-BspHI (GATCAATCATGATGGCTCCAACAAACGCAAC) and HervesR-KpnI (GTTCAAG GTACCTTGAATCCAATTAGCTATATTCTTACC) primers.

The resulting fragment was cloned into NcoI/KpnIdigested pBAD myc/HisA to generate pBADHvPCR1. The remaining Herves ORF (1,118 bp) was amplified using the HervesF-KpnI (CAAGGTACCTTGAACAAATTTGACATAGAGGATAAG) and HervesR-HindIII primers (TATCAAGCTTTGAACAAATTTGA CATAGAGGATAAG) and cloned into KpnI/HindIII digested pBADHvPCR1 to generate pBADHv1.

\section{Herves transposase purification}

Herves transposase was purified by His-tag purification as described [47]. pBADHv1-transformed LMG 194 $E$ coli cells were grown overnight at $30^{\circ} \mathrm{C}$ in LB media that contained carbenicillin $(100 \mathrm{mg} / \mathrm{ml})$. The overnight culture was diluted 1:100 in LB and carbenicillin $(100 \mathrm{mg} / \mathrm{ml})$ and grown at $30^{\circ} \mathrm{C}$ and $230 \mathrm{rpm}$ to an absorbance of 0.6 at $600 \mathrm{~nm}$. The cultures were then induced with $0.1 \% \mathrm{~L}$-arabinose and shaken at $16^{\circ} \mathrm{C}$ for $18 \mathrm{~h}$. The cells were harvested and washed by centrifugation with binding buffer $(0.5 \mathrm{M} \mathrm{NaCl}, 20 \mathrm{mM}$ Tris-Cl $\mathrm{pH} 7.9,10 \%$ glycerol, $10 \mathrm{mM}$ imidazole). The cells were lysed twice using a French press at 20,000 psi. The cell 
lysate was cleared by centrifugation and by passing through $0.45 \mu \mathrm{m}$ syringe filters. Cleared lysate was loaded onto Sepharose (Amersham/GE Healthcare, Piscataway, NJ) chromatography columns that were preequilibrated with $\mathrm{Ni}^{2+}$. The columns were washed with $10 \mathrm{ml}$ binding buffer and $6 \mathrm{ml}$ wash buffer $(0.5 \mathrm{M} \mathrm{NaCl}$, $20 \mathrm{mM}$ Tris-Cl pH 7.9, 10\% glycerol, $50 \mathrm{mM}$ imidazole). His-tagged Herves was eluted in five $1 \mathrm{ml}$ fractions of elution buffer $(0.5 \mathrm{M} \mathrm{NaCl}, 20 \mathrm{mM}$ Tris-Cl pH 7.9, 10\% glycerol, $200 \mathrm{mM}$ imidazole). The purified Herves transposase was dialyzed overnight in dialysis buffers $1(0.5$ $\mathrm{M} \mathrm{NaCl}, 20 \mathrm{mM}$ Tris base, $10 \%$ glycerol $\mathrm{pH} 8.0$ ) and 2 (0.5 M NaCl, $20 \mathrm{mM}$ Tris base, $2 \mathrm{mM}$ dithiothreitol (DTT), 25\% glycerol pH 8.0) for $3 \mathrm{~h}$ using a Slide-ALyzer dialysis cassette (Thermo Fisher Scientific, Waltham, MA). The dialyzed, purified Herves transposase was stored at $-80^{\circ} \mathrm{C}$.

\section{EMSAs}

The DNA fragment (100 nM) that we tested for transposase binding was end labeled using T4 polynucleotide kinase and ${ }^{32} \mathrm{P}$ ATP and purified on a Biospin $30 \mathrm{col}-$ umn (BioRad, Hercules, CA). The labeled DNA fragment (probe) was incubated at $4^{\circ} \mathrm{C}$ for 45 min with $1 \times$ EMSA binding buffer (16 mM Tris $\mathrm{pH} 8.0,0.2 \mu \mathrm{g}$ bovine serum albumin (BSA), $0.4 \mu \mathrm{g}$ T3 single-stranded oligo, $0.5 \mu \mathrm{g}$ poly(dI-dC), $1 \mathrm{mM}$ DTT, $150 \mathrm{mM} \mathrm{NaCl}$, $0.25 \%$ Triton X) and $850 \mathrm{nM}$ of Herves transposase. Specific and non-specific DNA fragments were used as specific and non-specific competitors, respectively (if applicable). The reaction was incubated with the probe for an additional $40 \mathrm{~min}$ at $4^{\circ} \mathrm{C}$. The non-specific competitors were $126 \mathrm{bp}$ gDNA fragment (E1) that flanks Hermes TE from Musca domestica and a 30 bp DNA oligo from Aedes aegypti $\beta 2$ tubulin. The EMSA reaction products were analyzed on a $5 \% \mathrm{TBE}$ polyacrylamide gel (Bio-Rad).

\section{DNase I protection assay}

DNA fragments (100 bp each) from the Herves-L and Herves-R ends, containing an EcoRV restriction site at the L-end or R-end, were cloned into pJET 1.2 (Fermentas/Thermo Fisher Scientific, Piscataway, NJ) to generate pL5'EcoRV, pL3'EcoRV, pR5'EcoRV, and pR3'EcoRV. The transferred and non-transferred strands from the Herves- $\mathrm{L}$ and Herves- $\mathrm{R}$ ends were selectively radiolabeled at one end by digesting pL5'EcoRV, pL3'EcoRV, pR5'EcoRV, and pR3'EcoRV with XhoI and EcoRV and labeling them with $\left[{ }^{32} \mathrm{P}\right]$ dATP using Klenow (NEB, Ipswich, MA). Herves transposase was allowed to bind to $100 \mathrm{nM}$ single end-labeled DNA fragment (probe) under the same binding conditions as in the EMSA. The optimal concentrations of transposase were determined empirically (Additional files 1 and 2). The DNA probe was subjected to DNase I digestion for 2 min at $4^{\circ} \mathrm{C}$. The reaction was stopped by adding stop solution $(92 \%$ ethanol, $0.7 \mathrm{M}$ ammonium acetate, $0.35 \mu \mathrm{g}$ tRNA) for $15 \mathrm{~min}$ in a dry ice/ethanol bath. DNA was extracted with phenol/chloroform and precipitated with ethanol. The reaction products were analyzed on a $10 \%$ denaturing polyacrylamide sequencing gel. The DNA sequencing kit 2.0 (USB) was used to construct a nucleotide ladder that was analyzed with the reaction products on the sequencing gel (Additional file 3).

\section{Additional material}

Additional file 1: DNase I protection of the Herves right (R) end.

Various concentrations of Herves transposase (as indicated) were tried to titrate for the optimum concentration for the protections assays for the Herves right end. Concentrations higher than $850 \mathrm{nM}$ (such as $1 \mu \mathrm{M}$ or $1.2 \mu \mathrm{M})$ or lower than $850 \mathrm{nM}(150 \mathrm{nM}, 300 \mathrm{nM}$ and $428 \mathrm{nM})$ produced non-specific protection of the probe or no protection at all, respectively. (a) $100 \mathrm{nM}$ or (b) $50 \mathrm{nM}$ and $100 \mathrm{nM}$ of the single-end-labeled Herves- $\mathrm{R}$ 1-100 bp fragment was incubated in absence (-) or presence of the transposase at various concentrations as indicated. ${ }^{32} \mathrm{P}$ indicates end of the probe that was labeled.

Additional file 2: DNase protection of the Herves left (L) end. Various concentrations of Herves transposase (as indicated) were tried to titrate for the optimum concentration for the protections assays for the Herves left end. Concentrations higher than $850 \mathrm{nM}$ (such as $1 \mu \mathrm{M}$ or $1.2 \mu \mathrm{M}$ ) or lower than $850 \mathrm{nM}(150 \mathrm{nM}, 300 \mathrm{nM}$ and $428 \mathrm{nM})$ produced non-specific protection of the probe or no protection at all, respectively. (a) $50 \mathrm{nM}$ or (b) $100 \mathrm{nM}$ of the single-end-labeled Herves-L 1-100 bp fragment was incubated in absence (-) or presence of the transposase at various concentrations as indicated. ${ }^{32} \mathrm{P}$ indicates end of the probe that was labeled.

Additional file 3: Figure 3with DNA ladder. The panel is identical to that shown in the left of Figure 3 but with a DNA ladder. The nucleotide positions were determined by the Sanger sequencing reactions shown in lanes $G$ and $A$.

\section{Acknowledgements}

This research was supported by PHS grants Al45741 and GM48102 to PWA and DAO, respectively, and by the Interdepartmental Graduate Program in Cell, Molecular and Developmental Biology at the University of California, Riverside.

\section{Author details}

'Interdepartmental Graduate Program in Cell, Molecular and Developmental Biology, University of California, Riverside, CA, USA. ${ }^{2}$ Institute of Integrative Genome Biology and Department of Entomology, University of California, Riverside, CA, USA. ${ }^{3}$ Department of Entomology and Institute for Bioscience and Biotechnology Research, University of Maryland, Rockville, MD, USA.

\section{Authors' contributions}

ASK performed the experiments and wrote early drafts of the manuscript. $\mathrm{RHH}$ provided technical expertise and assisted in experimental design. DAO participated in experimental design and edited the manuscript. PWA conceived the study, directed the experimentation and edited subsequent drafts of the manuscript. All authors read and approved the final manuscript.

\section{Competing interests}

The authors declare that they have no competing interests.

Received: 30 August 2010 Accepted: 20 June 2011

Published: 20 June 2011 


\section{References}

1. Jiang N, Bao Z, Zhang X, Eddy SR, Wessler SR: Pack-MULE transposable elements mediate gene evolution in plants. Nature 2004, 431:569-573.

2. Kidwell MG, Lisch D: Transposable elements as sources of genomic variation. In Mobile DNA II. Edited by: Craig NL, Craigie R, Gellert M, Lambowitz AM. Washington, DC: American Society for Microbiology Press; 2002:59-90.

3. Levis RW, Ganesan R, Houtchens K, Tolar LA, Sheen FM: Transposons in place of telomeric repeats at a Drosophila telomere. Cell 1993, 75:1083-1093.

4. Hurst GDD, Werren $\mathrm{JH}$ : The role of selfish genetic elements in eukaryotic evolution. Nat Rev Genet 2001, 2:597-606.

5. Dimitri $P$, Junakovic N: Revising the selfish DNA hypothesis: new evidence on accumulation of transposable elements in heterochromatin. Trends Genet 1999, 15:123-124.

6. Hua-Van A, Le Rouzic A, Maisonhaute C, Capy P: Abundance, distribution and dynamics of retrotransposable elements and transposons: similarities and differences. Cytogenet Genome Res 2005, 110:426-440.

7. Atkinson PW: Genetic engineering in insects of agricultural importance. Insect Biochem Mol Biol 2002, 32:1237-1242.

8. Smith RC, Walter MF, Hice RH, O'Brochta DA, Atkinson PW: Testis-specific expression of the $\beta 2$ tubulin promoter of Aedes aegypti and its application as a genetic sex-separation marker. Insect Mol Biol 2007, 16:61-71.

9. Grossman GL, Rafferty CS, Clayton JR, Stevens TK, Mukabayire O, Benedict MQ: Germline transformation of the malaria vector, Anopheles gambiae, with the piggyBac transposable element. Insect Mol Biol 2001, 10:597-604.

10. Catteruccia F, Nolan T, Loukeris TG, Blass C, Savakis C, Kafatos FC, Crisanti A: Stable germline transformation of the malaria mosquito Anopheles stephensi. Nature 2000, 405:959-962.

11. Jasinskiene N, Coates CJ, Benedict MQ, Cornel AJ, Rafferty CS, James AA Collins FH: Stable transformation of the yellow fever mosquito, Aedes aegypti, with the Hermes element from the housefly. Proc Natl Acad Sci USA 1998, 95:3743-3747.

12. Michel K, Stamenova A, Pinkerton AC, Franz G, Robinson AS, GariouPapalexiou A, Zacharopoulou A, O'Brochta DA, Atkinson PW: Hermesmediated germ-line transformation of the Mediterranean fruit fly, Ceratitis capitata. Insect Mol Biol 2001, 10:155-162.

13. Coates CJ, Jasinskiene N, Miyashiro L, James AA: Mariner transposition and transformation of the yellow fever mosquito, Aedes aegypti. Proc Natl Acad Sci USA 1998, 95:3748-3751.

14. O'Brochta DA, Atkinson PW, Lehane MJ: Transformation of Stomoxys calcitrans with a Hermes gene vector. Insect Mol Biol 2000, 9:531-538.

15. Miller LH, Sakai RK, Romans P, Gwadz RW, Kantoff P, Coon HG: Stable integration and expression of a bacterial gene in the mosquito, Anopheles gambiae. Science 1987, 237:779-781.

16. Kim W, Koo H, Richman AM, Seeley D, Vizioli J, Klocko AD, O'Brochta DA: Ectopic expression of a cecropin transgene in the human malaria vector mosquito Anopheles gambiae (Diptera: Culicidae): effects on susceptibility to Plasmodium. J Med Entomol 2004, 41:447-455.

17. Lombardo F, Lycett GJ, Lanfrancotti A, Coluzzi M, Arcà B: Analysis of apyrase $5^{\prime}$ upstream region validates improved Anopheles gambiae transformation technique. BMC Res Notes 2009, 2:24

18. Meredith JM, Basu S, Nimmo DD, Larget-Thiery I, Warr EL, Underhill A, McArthur CC, Carter V, Hurd H, Bourgouin C, Eggleston P: Site-specific integration and expression of an anti-malarial gene in transgenic Anopheles gambiae significantly reduces Plasmodium infections. PLoS One 2011, 6:e14587.

19. Papathanos PA, Windbichler N, Menichelli M, Burt A, Crisanti A: The vasa regulatory region mediates germline expression and maternal transmission of proteins in the malaria mosquito Anopheles gambiae: a versatile tool for genetic control strategies. BMC Mol Biol 2009, 10:65.

20. Saito K, Siomi MC: Small RNA-mediated quiescence of transposable elements in animals. Dev Cell 2010, 19:687-697.

21. Senti KA, Brennecke J: The piRNA pathway: a fly's perspective on the guardian of the genome. Trends Genet 2010, 26:499-509.

22. Arensburger P, Kim YJ, Orsetti J, Aluvihare C, O'Brochta DA, Atkinson PW: An active transposable element, Herves, from the African malaria mosquito Anopheles gambiae. Genetics 2005, 169:697-708.
23. Subramanian RA, Arensburger P, Atkinson PW, O'Brochta DA: Transposable element dynamics of the $h A T$ element Herves in the human malaria vector Anopheles gambiae s.s. Genetics 2007, 176:2477-2487.

24. Engels WR, Johnson-Schlitz DM, Eggleston WB, Sved J: High-frequency P element loss in Drosophila is homolog dependent. Cell 1990, 62:515-525.

25. Subramanian RA, Cathcart LA, Krafsur ES, Atkinson PW, O'Brochta DA: Hermes transposon distribution and structure in Musca domestica. $J$ Hered 2009, 100:473-480.

26. Galindo MI, Ladevèze $\mathrm{V}$, Lemeunier $\mathrm{F}$, Kalmes $\mathrm{R}$, Periquet $\mathrm{G}$, Pascual L: Spread of autonomous transposable element hobo in the genome of Drosophila melanogaster. Mol Biol Evol 1995, 12:723-734.

27. Craig NL: Mobile DNA: an introduction. In Mobile DNA II. Edited by: Craig NL, Craigie R, Gellert M, Lambowitz A. Washington, DC: ASM Press; 2002:3-11.

28. Coupland G, Plum C, Chatterjee S, Post A, Starlinger P: Sequences near the termini are required for transposition of the maize transposon $A c$ in transgenic tobacco plants. Proc Natl Acad Sci USA 1989, 86:9385-9388.

29. Li X, Harrell RA, Handler AM, Beam T, Hennessy K, Fraser MJ Jr: piggyBac internal sequences are necessary for efficient transformation of target genomes. Insect Mol Biol 2005, 14:17-30.

30. Liu D, Wang R, Galli M, Crawford NM: Somatic and germinal excision activities of the Arabidopsis transposon Tag1 are controlled by distinct regulatory sequences within Tag1. Plant Cell 2001, 13:1851-1863.

31. Liu D, Mack A, Wang R, Galli M, Belk J, Ketpura NI, Crawford NM: Functional dissection of the cis-acting sequences of Arabidopsis transposable element Tag1 reveals dissimilar subterminal sequence and minimal spacing requirements for transposition. Genetics 2001, 157:817-830.

32. Li X, Heinrich JC, Scott MJ: piggyBac-mediated transposition in Drosophila melanogaster: an evaluation of the use of constitutive promoters to control transposase gene expression. Insect Mol Biol 2001, 10:447-455.

33. Kunze $R$, Starlinger $P$ : The putative transposase of transposable element Ac from Zea mays $L$. interacts with subterminal sequences of Ac. EMBO J 1989, 8:3177-3185.

34. Urasakai A, Morvan G, Kawakami K: Functional dissection of the Tol2 transposable element identified the minimal cis-sequence and a highly repetitive sequence in the subterminal region essential for transposition. Genetics 2006, 174:639-649.

35. Atkinson $\mathrm{H}$, Chalmers $\mathrm{R}$ : Delivering the goods: viral and non-viral gene therapy systems and the inherent limits on cargo DNA and internal sequences. Genetica 2010, 138:485-498.

36. Yang G, Nagel DH, Feschotte C, Hancock CN, Wessler SR: Tuned for transposition: molecular determinants underlying the hyperactivity of a Stowaway MITE. Science 2009, 325:1391-1394.

37. Guynet C, Archard A, Hoang BT, Barabas O, Hickman AB, Dyda F, Chandler M: Resetting the site: redirecting integration of an insertion sequence in a predictable way. Molec Cell 2009, 34:612-619.

38. Mullins MC, Rio DC, Rubin GM: cis-acting DNA sequence requirements for P-element transposition. Genes Dev 1989, 3:729-738.

39. Cristancho MA, Gaitan AL: Isolation, characterization and amplification of simple sequence repeat loci in coffee. Crop Breed Appl Biotechnol 2008, 8:321-329.

40. Craigie R, Mizuuchi K: Site-specific recognition of the bacteriophage $\mathrm{Mu}$ ends by the MuA protein. Cell 1984, 39:387-394

41. Vos JC, Plasterk RHA: Tc1 transposase of Caenorhabditis elegans is an endonuclease with a bipartite DNA binding domain. EMBO J 1994, 13:6125-6132.

42. Colloms SD, van Luenen HGAM, Plasterk RHA: DNA binding activities of the Caenorhabditis elegans Tc3 transposase. Nucleic Acids Res 1994 22:5548-5554.

43. Mack AM, Crawford NM: The Arabidopsis TAG1 transposase has an Nterminal zinc finger DNA binding domain that recognizes distinct subterminal motifs. Plant Cell 2001, 13:2319-2331.

44. Warren WD, Atkinson PW, O'Brochta DA: The Hermes transposable element from the housefly, Musca domestica, is a short inverted repeattype element of the hobo, Ac, and Tam3 ( $h A T$ ) element family. Genetic Res Camb 1994, 64:87-97.

doi:10.1186/1759-8753-2-9

Cite this article as: Kahlon et al:: DNA binding activities of the Herves transposase from the mosquito Anopheles gambiae. Mobile DNA 2011 2:9. 